Honam Mathematical J. 34 (2012), No. 3, pp. 451-465

http://dx.doi.org/10.5831/HMJ.2012.34.3.451

\title{
COMPARISON OF CONTINUITIES IN DIGITAL TOPOLOGY
}

\author{
SiK LeE AND SANG-EON HAN
}

\begin{abstract}
Since there are several kinds of continuities of maps between digital spaces, the paper compares them, which can play an important role in digital topology and discrete geometry.
\end{abstract}

\section{Introduction}

Let $\mathbf{N}, \mathbf{Z}$ and $\mathbf{Z}^{n}$ be the sets of natural numbers, integers and points in the Euclidean $n \mathrm{D}$ space with integer coordinates, respectively. Digital topology including Khalimsky topology has been often used for studying various properties of objects in $\mathbf{Z}^{n}[2,4,5,6,7,8,9,10,11,12,13,14$, $15,16,17,18,19]$.

While many works in digital topology deal objects in $\mathbf{Z}^{n}$ with various types of topological structures and digital connectivity (or $k$-adjacency relations of $\mathbf{Z}^{n}$ ), Khalimsky topology recognizes objects in $\mathbf{Z}^{n}$ as Khalimsky $n \mathrm{D}$ subspaces. These approaches play important roles in computer graphics, image synthesis, image analysis, mathematical morphology and so forth.

In this paper we study an object in $\mathbf{Z}^{n}$ by using special kinds of neighborhoods induced from both the Khalimsky topological structure and graph theoretical tools. To be specific, for a set $X \subset \mathbf{Z}^{n}$ we study the subspace $\left(X, T_{X}^{n}\right)$ of the Khalimsky $n \mathrm{D}$ space $\left(\mathbf{Z}^{n}, T^{n}\right), n \geq 1$ $[7,10,14,15]$ and further, consider the subspace $\left(X, T_{X}^{n}\right)$ with one of the $k$-adjacency relations of $\mathbf{Z}^{n}$, denoted by $X_{n, k}$. In order to study $X_{n, k}:=\left(X, k, T_{X}^{n}\right)$, up to now several kinds of continuities are used, e.g. Khalimsky continuity combined with digital continuity [7], briefly

Received August 10, 2012. Accepted August 29, 2012.

2000 Mathematics Subject Classification. 54C20, 54C05, 54D05, 54F05, 68 U05.

Key words and phrases. digital topology, Khalimsky topology, digital continuity, digital isomorphism (homeomorphism).

This study was financially supported by Chonnam National University, 2011. The second author is the corresponding author of the paper. 
called $K D$ - $\left(k_{0}, k_{1}\right)$-continuity (see Definition 4$)$, and $\left(k_{0}, k_{1}\right)$-continuity (see Definition 5). Even though the $k$-adjacency of $X \subset \mathbf{Z}^{n}$ is not directly related to the Khalimsky topology [3], the notion gives us powerful benefits in studying objects $X \subset \mathbf{Z}^{n}$. The papers $[4,7,10,14]$ discusses limitations of a Khalimsky continuous map. More precisely, a Khalimsky continuous map only partially preserves digital connectivity and a geometric transformation. Owing to this shortage, several continuities of maps between Khalimsky spaces were developed $[4,7,10,15]$. Let DTC be the category of digital images and digitally $\left(k_{0}, k_{1}\right)$-continuous maps, $K D T C$ the category of spaces $X_{n, k}$ and $\mathrm{KD}-\left(k_{0}, k_{1}\right)$-continuous maps, and $C T C$ the category of spaces $X_{n, k}$ and $\left(k_{0}, k_{1}\right)$-continuous maps.

As mentioned above, a Khalimsky continuous map $f: X_{n_{0}, k_{0}} \rightarrow$ $Y_{n_{1}, k_{1}}$ need not preserve the $k_{0}$-connectedness of $X_{n_{0}, k_{0}}$ into the $k_{1^{-}}$ connectedness of $Y_{n_{1}, k_{1}}$ (see Remark 3.2). Besides, the connectedness in Khalimsky topology is different from the $k$-connectedness in digital topology [3]. These are the reasons why we study a set $X \subset \mathbf{Z}^{n}$ in $K D T C$ and $C T C$. Thus, by using these categories, we need to study objects in $\mathbf{Z}^{n}$ from the viewpoints of DTC, KDTC, and CTC. Each of these categories has intrinsic benefits for studying objects in $\mathbf{Z}^{n}$ from the viewpoint of digital topology.

This paper is organized as follows. Section 2 provides basic notions. Section 3 compares several continuities in digital topology and investigates their various properties which are extensions of the earlier papers in $[7,10]$. Section 4 provides some remarks and further works.

\section{Preliminaries}

A set $X \subset \mathbf{Z}^{n}$ with digital connectivity (or $k$-adjacency relations of $\left.\mathbf{Z}^{n}\right)$, denoted by $(X, k)$ and called a digital image, is usually considered in a quadruple $\left(\mathbf{Z}^{n}, k, \bar{k}, X\right)$, where $n \in \mathbf{N}, k$ represents an adjacency relation for $X$, and $\bar{k}$ represents an adjacency relation for $\mathbf{Z}^{n} \backslash X[16,18$, 19]. Thus, we can consider a set $X$ in $\left(\mathbf{Z}^{n}, k, 2 n, X\right)$ or $\left(\mathbf{Z}^{n}, 2 n, 3^{n}-1, X\right)$ with $k \neq 2 n$ except $(\mathbf{Z}, 2,2, X)$ owing to the digital connectivity paradox related to the digital Jordan curve theorem in [18]. However, in this paper we are not concerned with adjacencies among $n$-xels of $\mathbf{Z}^{n} \backslash X$.

As a generalization of the commonly used 4- and 8-adjacency of $\mathbf{Z}^{2}$ and further, 6-, 18-, and 26-adjacency of $\mathbf{Z}^{3}$ in [18, 19], $k$-adjacency relations of $\mathbf{Z}^{n}$ have been used for studying a set $X \subset \mathbf{Z}^{n}, n \geq 1$ [7] (see also $[5,6,7,8,9,10,11])$ : Let $m$ be a positive integer with $1 \leq m \leq n$. Two distinct points $p=\left(p_{i}\right)_{i \in[1, n]_{\mathbf{Z}}}$ and $q=\left(q_{i}\right)_{i \in[1, n]_{\mathbf{Z}}} \in \mathbf{Z}^{n}$ are called 
$k(m, n)$ (briefly, $k)$-adjacent according to $m$ if

- there are at most $m$ indices $i$ such that $\left|p_{i}-q_{i}\right|=1$; and

- for all other indices $i$ such that $\left|p_{i}-q_{i}\right| \neq 1, p_{i}=q_{i}$.

In this operator $k:=k(m, n)$ is the number of points $q$ which are $k$ adjacent to a given point $p$ according to the numbers $m$ and $n$ in $\mathbf{N}$, where " $:="$ means equal by definition. Indeed, this $k(m, n)$-adjacency is another presentation of the $k$-adjacency of $[5,8]$. Consequently, this operator leads to the representation of the $k$-adjacency relations of $\mathbf{Z}^{n}$ [9] (for more details, see [10]):

$$
k:=k(m, n)=\sum_{i=n-m}^{n-1} 2^{n-i} C_{i}^{n},
$$

where $C_{i}^{n}=\frac{n !}{(n-i) ! i !}$.

A set $X \subset \mathbf{Z}^{n}$ can be considered as a subspace $\left(X, T_{X}^{n}\right)$ induced from the Khalimsky $n \mathrm{D}$ space $\left(\mathbf{Z}^{n}, T^{n}\right)$ in [16]. Indeed, Khalimsky line topology on $\mathbf{Z}$ is induced from the subbase $\left\{[2 n-1,2 n+1]_{\mathbf{Z}} \mid n \in \mathbf{Z}\right\}$ [16]. Then we use the notation $(\mathbf{Z}, T)$. For $\{a, b\} \subset \mathbf{Z}$ with $a \leq b$, $[a, b]_{\mathbf{Z}}=\{a \leq n \leq b \mid n \in \mathbf{Z}\}$ is called a digital interval [2]. Besides, if $[a, b]_{\mathbf{Z}}$ is considered as a subspace $\left([a, b]_{\mathbf{Z}}, T_{[a, b]_{\mathbf{Z}}}\right)$ induced from the Khalimsky line space $(\mathbf{Z}, T)$, then it is called a Khalimsky interval. In this paper, we denote it $[a, b]_{\mathbf{Z}}$ instead of $\left([a, b]_{\mathbf{Z}}, T_{[a, b]_{\mathbf{Z}}}\right)$ if there is no confusion. Furthermore, the product topology on $\mathbf{Z}^{n}$ is derived from the Khalimsky line topology on $\mathbf{Z}, n \geq 2$. Then the topology on $\mathbf{Z}^{n}$ is called the Khalimsky (product) topology on $\mathbf{Z}^{n}$ and we use the notation $\left(\mathbf{Z}^{n}, T^{n}\right)$ called the Khalimsky $n D$ space [16]. In addition, a point $x=$ $\left(x_{i}\right)_{i \in[1, n]_{\mathbf{Z}}} \in \mathbf{Z}^{n}$ is open if all coordinates are odd, and closed if each of the coordinates is even [16]. These points are called pure and the other points in $\mathbf{Z}^{n}$ is called mixed. In all subspaces of $\left(\mathbf{Z}^{n}, T^{n}\right), n \geq 2$, of Figures 1-3 the symbols $\mathbf{\square}$, • and a jumbo dot mean a pure closed point, a mixed point, and a pure open point, respectively.

\section{Comparison of continuities in digital topology}

For a set $X \subset \mathbf{Z}^{n}$ let us consider a subspace $\left(X, T_{X}^{n}\right)$ induced from the Khalimsky $n \mathrm{D}$ space $\left(\mathbf{Z}^{n}, T^{n}\right)$. Furthermore, it is meaningful to consider $\left(X, T_{X}^{n}\right)$ with $k$-adjacency of $\mathbf{Z}^{n}$. This approach makes helpful to find some important information of $\left(X, T_{X}^{n}\right)$ in digital topology such as various continuities, homeomorphisms and so forth. 
Definition 1. [7] Considering a topological space $\left(X, T_{X}^{n}\right)$ with $k$ adjacency, we call it a space with $k$-adjacency (or a space) and we denote it $\left(X, k, T_{X}^{n}\right)$, briefly $X_{n, k}$.

In order to find some important features of $X_{n, k}$ and to establish several kinds of mathematical categories, we need to recall terminology as follows. For a set with $k$-adjacency (or a digital image) $(X, k)$ in $\mathbf{Z}^{n}$, two points $x, y \in X$ with $x \neq y$ are called $k$-connected if there is a $k$-path $f:[0, m]_{\mathbf{Z}} \rightarrow X$ whose image is an injective sequence $\left(x_{i}\right)_{i \in[0, m]_{\mathbf{Z}}} \subset X$ from the set of points $\left\{f(0)=x_{0}=x, f(1)=x_{1}, \cdots, f(m)=x_{m}=y\right\}$ such that $x_{i}$ and $x_{i+1}$ are $k$-adjacent, $i \in[0, m-1]_{\mathbf{Z}}$. The number $m$ is called the length of this $k$-path [19]. For an adjacency relation $k$ of (2.1), a simple $k$-path in $X \subset \mathbf{Z}^{n}$ is a sequence $\left(x_{i}\right)_{i \in[0, m]_{\mathbf{Z}}} \subset X$ such that $x_{i}$ and $x_{j}$ are $k$-adjacent if and only if either $j=i+1$ or $i=j+1$ [18].

For a space $X_{n, k}:=\left(X, k, T_{X}^{n}\right)$ we consider the notions of digital and Khalimsky topological $k$-neighborhoods for developing the notions of $\mathrm{KD}-\left(k_{0}, k_{1}\right)$ - and $\left(k_{0}, k_{1}\right)$-continuities of Definitions 4 and 5 , as follows.

Definition 2. [7](see also $[4,10])$ For $X_{n, k}:=\left(X, k, T_{X}^{n}\right), x, y \in X$, and $\varepsilon \in \mathbf{N}$, we consider the following.

(1) A subset $V$ of $X$ is called a neighborhood of $x$ if there exists an open set $O_{x} \in T_{X}^{n}$ such that $x \in O_{x} \subseteq V$.

(2) By $N_{k}(x, \varepsilon)$ we denote the subset of $X$

$$
\left\{y \in X: l_{k}(x, y) \leq \varepsilon\right\} \cup\{x\}, \varepsilon \in \mathbf{N},
$$

where $l_{k}(x, y)$ is the length of a shortest simple $k$-path from $x$ to $y$ in $X$ [5]. Besides, we say that $l_{k}(x, y)=\infty$ if there is no $k$-path from $x$ to $y$. The set $N_{k}(x, \varepsilon)$ is called a digital $k$-neighborhood of $x$ with radius $\varepsilon$.

(3) If the above set $N_{k}(x, \varepsilon)$ is a Khalimsky topological neighborhood of $x$ in $\left(X, T_{X}^{n}\right)$, then this set is called a $k$-neighborhood of $x$ with radius $\varepsilon \in \mathbf{N}$. In this paper, we use the notation $N_{k}^{*}(x, \varepsilon)$.

By Definition 2(2), if for a digital image $(X, k)$, a point $x \in X$ is not $k$-connected with any point in $X$, then we observe $N_{k}(x, \varepsilon)=\{x\}$ for any $\varepsilon \in \mathbf{N}$.

In $X_{n, k}$, we obviously obtain the following because $N_{3^{n}-1}(x, 1)$ always contains an open set $O_{x} \in T_{X}^{n}$ satisfying $x \in O_{x} \subset N_{3^{n}-1}(x, 1)$.

Proposition 3.1. For each point $x \in X_{n, k}, N_{k}(x, 1)=N_{k}^{*}(x, 1)$ if $k=3^{n}-1, n \in \mathbf{N}$.

By the use of the digital $k$-neighborhood of Definition 2 (2), motivated by the digital continuity in $[2,19]$, the digital $\left(k_{0}, k_{1}\right)$-continuity has been 
represented mathematically because any point in $(X, k)$ has $N_{k}(x, 1)$ in $X$.

Definition 3. [5] (see also [8]) Let $\left(X, k_{0}\right)$ and $\left(Y, k_{1}\right)$ be digital images in $\mathbf{Z}^{n_{0}}$ and $\mathbf{Z}^{n_{1}}$, respectively. We say that a function $f: X \rightarrow Y$ is digitally $\left(k_{0}, k_{1}\right)$-continuous if $f\left(N_{k_{0}}(x, 1)\right) \subset N_{k_{1}}(f(x), 1)$.

Let us consider a digital topological category, denoted by $D T C$, consisting of two things [5] (see also [7]):

- A set of objects $(X, k)$ in $\mathbf{Z}^{n}$;

- DTC has digitally $\left(k_{0}, k_{1}\right)$-continuous maps as morphisms.

For an adjacency relation $k$, we recall that a simple closed $k$-curve with $l$ elements in $X \subset \mathbf{Z}^{n}$ is the image of a digitally $(2, k)$-continuous function $f:[0, l-1]_{\mathbf{z}} \rightarrow X$ such that $f(i)$ and $f(j)$ are $k$-adjacent if and only if $i=j \pm 1(\bmod l)[18]$ and is denoted by $S C_{k}^{n, l}$ which is assumed to be a sequence $\left(c_{i}\right)_{i \in[0, l-1] \mathbf{Z}}$, where $f(i)=c_{i}[5]$.

For two digital images $\left(X, k_{0}\right)$ in $\mathbf{Z}^{n_{0}}$ and $\left(Y, k_{1}\right)$ in $\mathbf{Z}^{n_{1}}$, a map $h$ : $\left(X, k_{0}\right) \rightarrow\left(Y, k_{1}\right)$ is called a $\left(k_{0}, k_{1}\right)$-homeomorphism if $h$ is $\left(k_{0}, k_{1}\right)$ continuous and bijective and further, $h^{-1}: Y \rightarrow X$ is $\left(k_{1}, k_{0}\right)$-continuous in [2]. Then we use the notation $X \approx_{\left(k_{0}, k_{1}\right)} Y$. If $n_{0}=n_{1}$ and $k_{0}=k_{1}$, then we call it a $k_{0}$-homeomorphism [2]. Since a digital image can be recognized to be both a digital $k$-graph and a simplicial complex in [6], we may use a $\left(k_{0}, k_{1}\right)$-isomorphism instead of a $\left(k_{0}, k_{1}\right)$-homeomorphism in digital topology [8].

Remark 3.2. [7] (see also $[4,10]$ ) A Khalimsky continuous map $f: X_{n_{0}, k_{0}} \rightarrow Y_{n_{1}, k_{1}}$ need not preserve the $k_{0}$-connectedness into the $k_{1}$-connectedness.

Since the preservation of the $k_{0}$-connectedness of $X_{n_{0}, k_{0}}$ into the $k_{1^{-}}$ connectedness of $Y_{n_{1}, k_{1}}$ is so meaningful for the study of some objects in lattice based approach, we need the following notion.

Definition 4. [7] (see also [10]) A function $f: X_{n_{0}, k_{0}} \rightarrow Y_{n_{1}, k_{1}}$ is said to be Khalimsky continuous with digital $\left(k_{0}, k_{1}\right)$-continuity (briefly, $K D$ - $\left(k_{0}, k_{1}\right)$-continuous) at a point $x \in X_{n_{0}, k_{0}}$ if

(1) $f$ is Khalimsky continuous at the point $x$ and

(2) $f$ is digitally $\left(k_{0}, k_{1}\right)$-continuous at the point $x \in X_{n_{0}, k_{0}}$.

Furthermore, we say that a map $f: X_{n_{0}, k_{0}} \rightarrow Y_{n_{1}, k_{1}}$ is $K D-\left(k_{0}, k_{1}\right)$ continuous if it is $\mathrm{KD}-\left(k_{0}, k_{1}\right)$-continuous at every point $x \in X_{n_{0}, k_{0}}$.

In Definition 4, if $n_{0}=n_{1}$ and $k_{0}=k_{1}$, then we call the map $f$ a $K D$ $k_{0}$-continuous map. By Remark 3.2 , the current $\mathrm{KD}$ - $\left(k_{0}, k_{1}\right)$-continuity 
plays an important role in studying objects $X \subset \mathbf{Z}^{n}$ from the viewpoint of digital topology. Reminding Remark 3.2, we now need to further explain the reason why the $\mathrm{KD}-\left(k_{0}, k_{1}\right)$-continuity is meaningful for the study of a Khalimsky topological space (see Remarks 3.2 and 3.3, and Theorem 3.4).

The Khalimsky continuity of Definition 4(1) means the general topological continuity between Khalimsky topological spaces. In general, we can observe that none of the conditions (1) and (2) of Definition 4 implies the other (see Remark 3.3), as follows.

Remark 3.3. [7, 10, 14, 15] None of Khalimsky continuity and digital $\left(k_{0}, k_{1}\right)$-continuity of Definition 4 implies the other. Precisely, by Remark 3.2, it turns out that the condition (1) of Definition 4 need not lead to the condition (2) of Definition 4. Meanwhile, in general, since $N_{k}(x, \varepsilon)$ is different from a Khalimsky topological $k$-neighborhood $N_{k}^{*}(x, \varepsilon)$ or an open set in $\left(X, T_{X}^{n}\right)$, the condition (2) of Definition 4 need not imply the condition (1) of Definition 4.

For a space $X_{n, k}$ and its subspace $A_{n, k}$, we call $\left((X, A), k, T_{(X, A)}^{n}\right)$ a space pair with $k$-adjacency, denoted by $(X, A)_{n, k}$. For two space pairs $(X, A)_{n_{0}, k_{0}}$ and $(Y, B)_{n_{1}, k_{1}}$, we say that $f:(X, A)_{n_{0}, k_{0}} \rightarrow(Y, B)_{n_{1}, k_{1}}$ is $\mathrm{KD}-\left(k_{0}, k_{1}\right)$-continuous if $f: X_{n_{0}, k_{0}} \rightarrow Y_{n_{1}, k_{1}}$ is $\mathrm{KD}-\left(k_{0}, k_{1}\right)$-continuous and $f\left(A_{n_{0}, k_{0}}\right) \subset B_{n_{1}, k_{1}}[7]$.

The spaces $(\mathbf{Z}, T)$ and $\left(\mathbf{Z}^{n}, T^{n}\right)$ are connected in Khalimsky product topology. Furthermore, in $(\mathbf{Z}, T), 2$-connectedness is clearly equivalent to connectedness. Let us now examine the general cases as follows.

Theorem 3.4. For $X_{n, k}, n \geq 2$, we obtain the following.

(a) $k$-connectedness implies connectedness if $k=2 n$.

(b) The assertion of (a) need not hold if $k \neq 2 n$.

(c) Connectedness implies $k$-connectedness if $k=3^{n}-1$.

(d) The assertion of (c) need not hold if $k \neq 3^{n}-1$.

Proof: (a) Consider any points $x, y \in X$ such that $x$ and $y$ are $2 n$ connected with $x \neq y$ (for instance, see the points $x, y, z, w$ of $X$ in Figure 1(a)). Then there is a sequence $\left(x_{i}\right)_{i \in[0, m]_{\mathbf{Z}}} \subset X$ such that $x_{0}=x$, $x_{m}=y$, and both of $x_{i}$ and $x_{i+1}$ are $2 n$-adjacent, $i \in[0, m-1]_{\mathbf{Z}}$. Then, for any $i \in[0, m-1]_{\mathbf{Z}}$ the two points $x_{i}$ and $x_{i+1}$ are always in a same component of the Khalimsky $n \mathrm{D}$ space (see Figure 1(a)). Thus $X_{n, k}$ cannot be a union of non-empty open subsets $A$ and $B$ of $X_{n, k}$ such that $A \cap B=\emptyset$. 
(b) In $\mathbf{Z}^{2}$, consider the topological space $\left(W, T_{W}^{2}\right)$, where $W=\left\{w_{1}, w_{2}\right.$, $\left.w_{3}, w_{4}\right\} \subset \mathbf{Z}^{2}$ in Figure $1(\mathrm{~d})$. While the points $w_{2}$ and $w_{3}$ are 8connected, these points are in different components $\left\{w_{1}, w_{2}\right\}$ and $\left\{w_{3}, w_{4}\right\}$ because $\left\{w_{1}, w_{2}\right\}$ and $\left\{w_{3}, w_{4}\right\}$ are open sets in $T_{W}^{2}$.

In $\mathbf{Z}^{3}$, consider $Y:=\left\{y_{i}\right\}_{i \in[0,3]_{\mathbf{Z}}}$ in Figure 1(b). While $Y$ is 18connected, it is not connected in $\left(Y, T_{Y}^{2}\right)$ because $Y$ consists of two components $\left\{y_{0}\right\}$ and $\left\{y_{1}, y_{2}, y_{3}\right\}$.

In $\mathbf{Z}^{3}$, consider another space $Z:=\left\{z_{i}\right\}_{i \in[0,3] \mathbf{z}}$ in Figure $1(\mathrm{c})$. While $Z$ is 26 -connected, it is not connected because $Z$ consists of two components $\left\{z_{0}\right\}$ and $\left\{z_{1}, z_{2}, z_{3}\right\}$.

In general, in $\mathbf{Z}^{n}, n \geq 4$, by the same method as the above $2 D$ - and $3 D$-cases, we can prove that a $k$-connected space $X_{n, k}, k \neq 2 n$, need not be connected in Khalimsky product topology.

(c) Due to the Khalimsky product topology of $X_{n, k}$, connectedness of $X_{n, k}$ obviously implies $k$-connectedness of $X_{n, k}$ if $k=3^{n}-1$.

(d) If $k \neq 3^{n}-1$, then connectedness need not imply $k$-connectedness. For instance, consider the set $D:=\left\{d_{0}=(0,0,0), d_{1}=(1,1,1)\right\}$ with $\left(D, T_{D}^{3}\right)$. While $D$ is connected because $T_{D}^{3}=\left\{\emptyset, D,\left\{d_{1}\right\}\right\}$, it cannot be 18-connected. Furthermore, in $\mathbf{Z}^{n}$, we can consider a general case of the above mentioned set $D$ with $\left(D, T_{D}^{n}\right), n \geq 3$.

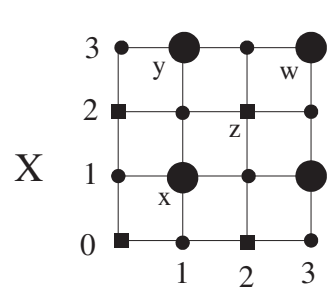

(a)

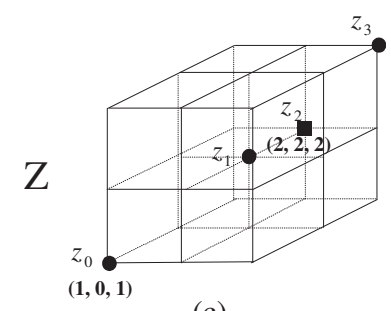

(c)

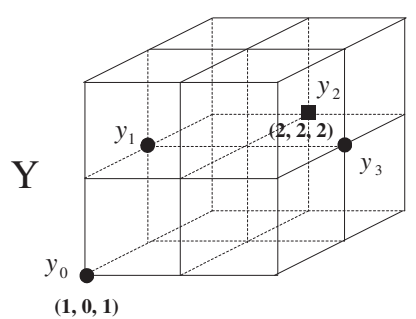

(b)

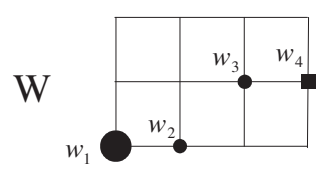

(d)

Figure 1. Comparison connectedness with $k$-connectedness. 
By Remarks 3.2 and 3.3, and Theorem 3.4, we observe that KD$\left(k_{0}, k_{1}\right)$-continuity gives some benefits in studying a space $X_{n, k}$. Thus let us consider a KD-topological category, denoted by KDTC, consisting of two things $[7,10]$ :

- A set of objects $X_{n, k}$;

- KDTC has KD- $\left(k_{0}, k_{1}\right)$-continuous maps as morphisms.

Even though $K D T C$ is so useful for studying $X_{n, k}$, it need not be sufficient in digital topology. For instance, consider two spaces $\left(X_{i}\right)_{2,8}$, $i \in\{1,2\}$, where $X_{i}$ is considered in Figure 3(a). Then, while a map $f: X_{1} \rightarrow X_{2}$ given by $f\left(x_{i}\right)=c_{i}, i \in[0,3]_{\mathbf{Z}}$ is digitally 8-continuous, $f$ cannot be KD-8-continuous at two points $x_{0}$ and $x_{2}$.

Furthermore, consider the spaces $\left(Z_{i}\right)_{3,26}, i \in\{1,2\}$, where the set $Z_{i}$ is considered in Figure 3(c). Then, while the map $h:\left(Z_{2}\right)_{3,26} \rightarrow\left(Z_{1}\right)_{3,26}$ for which $h\left(w_{i}\right)=z_{i}, i \in[0,3]_{\mathbf{Z}}$, is digitally 26-continuous, it cannot be a KD-26-continuous map at two points $w_{0}$ and $w_{2}$. These examples show the need of another continuity in digital topology. Thus we establish another continuity which is different from $\mathrm{KD}-\left(k_{0}, k_{1}\right)$-continuity as follows.

Definition 5. [7] (see also $[12,15]$ ) For two spaces $X_{n_{0}, k_{0}}:=X$ and $Y_{n_{1}, k_{1}}:=Y$ a function $f: X \rightarrow Y$ is said to be $\left(k_{0}, k_{1}\right)$-continuous at a point $x \in X$ if $f\left(N_{k_{0}}^{*}(x, r)\right) \subset N_{k_{1}}^{*}(f(x), s)$, where the number $r$ is the least element of $\mathbf{N}$ such that $N_{k_{0}}^{*}(x, r)$ contains an open set including the point $x$ and $s$ is the least element of $\mathbf{N}$ such that $N_{k_{1}}^{*}(f(x), s)$ contains an open set including the point $f(x)$.

Furthermore, we say that a map $f: X \rightarrow Y$ is $\left(k_{0}, k_{1}\right)$-continuous if the map $f$ is $\left(k_{0}, k_{1}\right)$-continuous at every point $x \in X$.

By Proposition 3.1, for any point $x \in X_{n, k}$, if $k=3^{n}-1$, then we obtain the following.

Corollary 3.5. If $k_{i}=3^{n_{i}}-1, i \in\{1,2\}$, then $\left(k_{0}, k_{1}\right)$-continuity is equivalent to digital $\left(k_{0}, k_{1}\right)$-continuity.

By the similar method as Remark 3.3, we obtain the following.

Remark 3.6. [7] (see also $[4,14,15])$ None of $\left(k_{0}, k_{1}\right)$-continuity and Khalimsky continuity implies the other.

We observe some properties of $\left(k_{0}, k_{1}\right)$-continuity:

Remark 3.7. (1) We observe that the map $f \pm g: X_{n_{0}, k_{0}} \rightarrow Y_{n_{1}, k_{1}}$ need not be a $\left(k_{0}, k_{1}\right)$-continuous map, where the componentwise ' \pm ' operations are typically determined because $f(x)$ and $g(x)$ are points 
in $\mathbf{Z}^{n_{1}}$. Besides, we observe that the composite property of $\left(k_{0}, k_{1}\right)$ continuous maps holds.

(2) We observe that $\left(k_{0}, k_{1}\right)$-continuity need not have the restriction property. For instance, consider a map $f: X^{\prime} \rightarrow Y:=\left\{y_{i}\right\}_{i \in[0,3]_{\mathbf{z}}}$ in Figure 2 given by

$f\left(\left\{x_{0}, x_{1}, x_{2}, x_{3}, x_{6}, d_{1}, d_{2}\right\}\right)=\left\{y_{0}\right\}, f\left(x_{4}\right)=y_{2}, f\left(x_{5}\right)=y_{1}$, and $f\left(\left\{x_{7}, x_{8}\right\}\right)=\left\{y_{3}\right\}$,

where $X^{\prime}=X \cup D, X=\left\{x_{i}\right\}_{i \in[0,8]_{\mathbf{z}}}$ and $D=\left\{d_{1}, d_{2}\right\}$. Consider $X_{2,4}^{\prime}$ and $Y_{2,4}$. Then $f: X_{2,4}^{\prime} \rightarrow Y_{2,4}$ is a $(4,4)$-continuous map. Let us now consider a restriction map $f$ on $X_{2,4}$, briefly $\left.f\right|_{X_{2,4}}$. Then, since the smallest open set containing the point $x_{0}$ is the set $\left\{x_{i}\right\}_{i \in[0,5] \mathbf{Z}}$, there is no $N_{4}^{*}\left(x_{0}, \varepsilon\right)$ for any $\varepsilon \in \mathbf{N}$ because $X_{2,4}$ is not 4-connected. Thus $\left.f\right|_{X_{2,4}}$ cannot be $(4,4)$-continuous.

Theorem 3.8. None of $K D$ - $\left(k_{0}, k_{1}\right)$-continuity and $\left(k_{0}, k_{1}\right)$-continuity implies the other. In other words,

(1) $\mathrm{KD}$ - $\left(k_{0}, k_{1}\right)$-continuity need not imply $\left(k_{0}, k_{1}\right)$-continuity.

(2) $\left(k_{0}, k_{1}\right)$-continuity need not lead to $K D$ - $\left(k_{0}, k_{1}\right)$-continuity.

Proof: (1) Consider the restriction map $\left.f\right|_{X_{2,4}}$ in Remark 3.7. While the map $\left.f\right|_{X_{2,4}}: X_{2,4} \rightarrow Y_{2,4}$ is KD-(4,4)-continuous, it cannot be $(4,4)$ continuous (see Remark 3.6).

(2) (a) Consider a map $f: A_{2,4} \rightarrow Y$ given by $f\left(a_{1}\right)=1$ and $f\left(a_{2}\right)=$ 2, where $A=\left\{a_{1}=(0,1), a_{2}=(1,1)\right\}$ and $Y=\{1,2\} \subset \mathbf{Z}$. Then, while the map $f$ is $(4,2)$-continuous, it cannot be Khalimsky continuous at the point $a_{1}$ because $\{1\} \in T_{Y}$ and $\left\{a_{1}\right\} \notin T_{A}^{2}$. Thus $f$ cannot be KD-(4,2)-continuous.

(b) Consider a map $f:\left(X_{1}\right)_{2,8} \rightarrow\left(X_{2}\right)_{2,8}$ given by $f\left(x_{i}\right)=c_{i}, i \in$ $[0,3]_{\mathbf{Z}}$, where $X_{j}, j \in\{1,2\}$, is the set in Figure $3(\mathrm{a})$. Then, while the map $f$ is 8 -continuous, it cannot be KD-8-continuous at the points $x_{0}$ and $x_{2}$ because the smallest open sets containing the points $x_{0}$ and $x_{2}$ are the sets $\left\{x_{0}, x_{1}, x_{3}\right\}$ and $\left\{x_{1}, x_{2}, x_{3}\right\}$, respectively.

In view of Theorem 3.8, we now have a mathematical category, denoted by $C T C$, consisting of two things:

- A set of objects $X_{n, k}$;

- $C T C$ has $\left(k_{0}, k_{1}\right)$-continuous maps as morphisms.

Let us now establish several homeomorphisms in digital topology.

Definition 6. [7] (see also [10]) In KDTC, a function $f: X_{n_{0}, k_{0}} \rightarrow$ $Y_{n_{1}, k_{1}}$ is said to be a $K D$ - $\left(k_{0}, k_{1}\right)$-homeomorphism if

(1) the map $f$ is bijective, and 


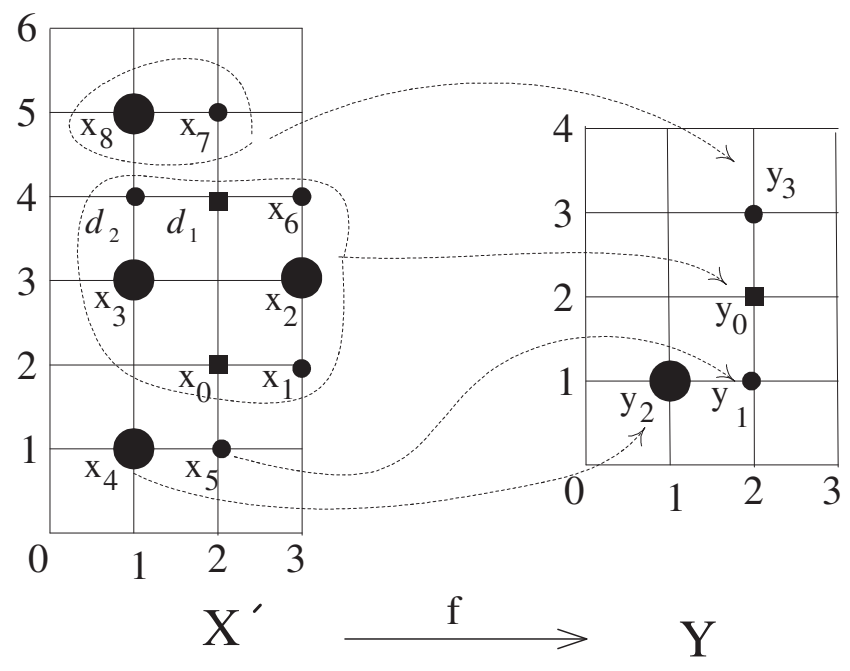

FIGURE 2. Non-restriction property of $\left(k_{0}, k_{1}\right)$-continuity.

(2) the map $f$ is a $K D$ - $\left(k_{0}, k_{1}\right)$-continuous map and further, $f^{-1}$ is a $K D$ - $\left(k_{1}, k_{0}\right)$-continuous map.

In this case we say that $X_{n_{0}, k_{0}}$ is $K D$ - $\left(k_{0}, k_{1}\right)$-homeomorphic to $Y_{n_{1}, k_{1}}$.

We now say that properties which when possessed by a space in $\mathbf{Z}^{n}$ are also possessed by $\left(k_{0}, k_{1}\right)$-, and $\mathrm{KD}$ - $\left(k_{0}, k_{1}\right)$-homeomorphisms are called $\left(k_{0}, k_{1}\right)$-, and $K D$ - $\left(k_{0}, k_{1}\right)$-homeomorphic properties, respectively.

Example 3.9. (1) In KDTC, let us consider two sets $X_{1}:=\left\{x_{i}\right\}_{i \in[0,3] \mathbf{z}}$ and $X_{2}:=\left\{c_{i}\right\}_{i \in[0,3]_{\mathbf{Z}}}$ in Figure 3(a). Then $T_{X_{1}}^{2}=\left\{\emptyset, X_{1},\left\{x_{1}\right\},\left\{x_{3}\right\},\left\{x_{0}, x_{1}, x_{3}\right\},\left\{x_{1}, x_{2}, x_{3}\right\},\left\{x_{1}, x_{3}\right\}\right\}$ and $T_{X_{2}}^{2}$ is essential to the discrete topological structure of $X_{2}$. While the two digital images $\left(X_{1}, 8\right)$ and $\left(X_{2}, 8\right)$ are 8-isomorphic to each other in DTC, $\left(X_{1}\right)_{2,8}$ and $\left(X_{2}\right)_{2,8}$ cannot be KD-8-homeomorphic to each other. To be specific, let us consider a map $h: X_{2} \rightarrow X_{1}$ given by $h\left(c_{i}\right)=x_{i}, i \in[0,3]_{\mathbf{z}}$. Then, while $h$ is a KD-8-continuous bijection, $h^{-1}$ cannot be KD-8-continuous at the points $x_{0}$ and $x_{2}$.

(2) Consider three sets $Y_{1}:=\left\{x_{i}\right\}_{i \in[0,5]_{\mathbf{Z}}}, Y_{2}:=\left\{y_{i}\right\}_{i \in[0,5]_{\mathbf{Z}}}$, and $Y_{3}:=\left\{c_{i}\right\}_{i \in[0,5] \mathbf{z}}$ in Figure 3(b). While the digital image $\left(Y_{i}, 18\right)$ is 18-isomorphic to $\left(Y_{j}, 18\right)$ in DTC, where $i, j \in\{1,2,3\},\left(Y_{i}\right)_{3,18}$ cannot be KD-18-homeomorphic to $\left(Y_{j}\right)_{3,18}$ if $i \neq j$. To be specific, let us examine two points $x_{2}$ and $x_{4}$ in $\left(Y_{1}\right)_{3,18}$. While the point $x_{i}$ has the 
smallest open set $O_{x_{i}}=N_{18}^{*}\left(x_{i}, 2\right)$ in $\left(Y_{1}\right)_{3,18}$, where $i \in\{2,4\}$ and $O_{x_{i}}$ is an open set containing the point $x_{i}$, the other point $x_{i}$ has the smallest open set $O_{x_{i}}=N_{18}^{*}\left(x_{i}, 1\right), i \in\{0,1,3,5\}$. Meanwhile, in $\left(Y_{2}\right)_{3,18}$, the point $y_{0}$ has the smallest open set $O_{y_{0}}$ containing the point $y_{0}$ such that $O_{y_{0}}=N_{18}^{*}\left(y_{0}, 2\right)$, and the other point $y_{i}$ has the smallest open set $O_{y_{i}}$ containing the point $y_{i}$ such that $O_{y_{i}}=N_{18}^{*}\left(y_{i}, 1\right), i \in\{1,2,3,4,5\}$. Thus $h:\left(Y_{2}\right)_{3,18} \rightarrow\left(Y_{1}\right)_{3,18}$ given by $h\left(y_{i}\right)=x_{i+2(\bmod 6)}, i \in[0,5]_{\mathbf{Z}}$, is a KD-18-continuous bijection, $h^{-1}$ cannot be KD-18-continuous, which means that there is no KD-18-homeomorphism between $\left(Y_{1}\right)_{3,18}$ and $\left(Y_{2}\right)_{3,18}$.

Besides, since $\left(Y_{3}\right)_{3,18}$ is essential to the discrete topological space of $Y_{3}$, neither $\left(Y_{1}\right)_{3,18}$ nor $\left(Y_{2}\right)_{3,18}$ is KD-18-homeomorphic to $\left(Y_{3}\right)_{3,18}$.

(3) Consider two sets $Z_{1}:=\left\{z_{i}\right\}_{i \in[0,3]_{\mathbf{z}}}$ and $Z_{2}:=\left\{w_{i}\right\}_{i \in[0,3] \mathbf{z}}$ in Figure 3(c). While the two digital images $\left(Z_{1}, 26\right)$ and $\left(Z_{2}, 26\right)$ are 26 isomorphic in DTC, $\left(Z_{1}\right)_{3,26}$ and $\left(Z_{2}\right)_{3,26}$ cannot be $K D$-26-homeomorphic to each other in KDTC. To be specific, let us consider a map $h$ : $\left(Z_{1}\right)_{3,26} \rightarrow\left(Z_{2}\right)_{3,26}$ given by $h\left(z_{i}\right)=w_{i}, i \in[0,3]_{\mathbf{Z}}$. While $T_{Z_{1}}^{3}$ is essential to the discrete topological structure of $Z_{1}$,

$T_{Z_{2}}^{3}=\left\{\emptyset, Z_{2},\left\{w_{1}\right\},\left\{w_{3}\right\},\left\{w_{1}, w_{2}, w_{3}\right\},\left\{w_{0}, w_{1}, w_{3}\right\},\left\{w_{1}, w_{3}\right\}\right\}$.

Thus $h$ is a KD-26-continuous bijection, $h^{-1}$ cannot be KD-26-continuous at the two points $w_{0}$ and $w_{2}$.

(a)
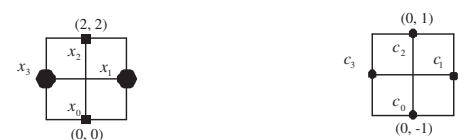

$\mathbf{X}_{1}$

$\mathbf{X}_{2}$

(b)
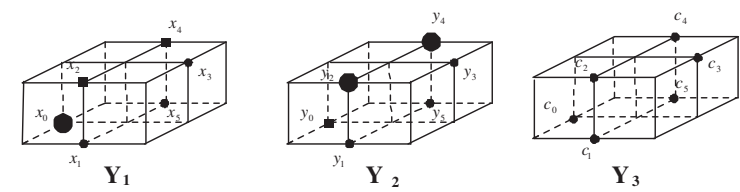

$\mathbf{Y}_{3}$

(c)

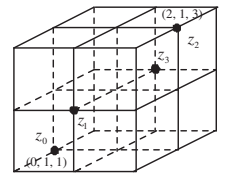

$\mathrm{Z}_{1}$

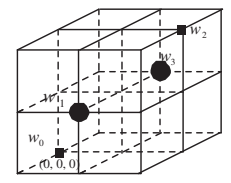

$\mathbf{Z}_{2}$

FiguRE 3 . Various spaces related to both $k$-isomorphism and KD- $k$-homeomorphism partially used in $[7,10]$. 
In view of Example 3.9, we obtain the following.

Theorem 3.10. (1) In KDTC, there are two types of simple closed 8curves with four elements in $\left(\mathbf{Z}^{2}, T^{2}\right)$ up to KD-8-homeomorphism such as $\left(X_{1}\right)_{2,8}$ and $\left(X_{2}\right)_{2,8}$ in Figure $3($ a).

(2) In KDTC, there are three types of simple closed 18-curves with six elements in $(2 \times 2 \times 1)$-form of $\left(\mathbf{Z}^{3}, T^{3}\right)$ up to KD-18-homeomorphism such as $\left(Y_{1}\right)_{3,6},\left(Y_{2}\right)_{3,6}$, and $\left(Y_{3}\right)_{3,6}$ in Figure $3(b)$.

(3) In KDTC, there are two types of simple closed 26-curves with four elements in $(2 \times 2 \times 2)$-form of $\left(\mathbf{Z}^{3}, T^{3}\right)$ up to KD-26-homeomorphism such as $\left(Z_{i}\right)_{3,26}$ in Figure $3(c), i \in\{1,2\}$.

(4) In KDTC, as a generalization of both $\left(X_{i}\right)_{2,8}$ and $\left(Z_{i}\right)_{3,26}, i \in$ $\{1,2\}$, in Figure 3, we observe that there are two types of simple closed $\left(3^{n}-1\right)$-curves with four elements in $\underbrace{(2 \times \cdots \times 2)}_{n \text {-times }}$-form of $\left(\mathbf{Z}^{n}, T^{n}\right)$ up to $K D$ - $\left(3^{n}-1\right)$-homeomorphism, $n \geq 4$.

Unlike various properties of $\mathrm{KD}-\left(k_{0}, k_{1}\right)$-continuity above mentioned, $\left(k_{0}, k_{1}\right)$-continuity has some intrinsic features which are similar to those of digital $\left(k_{0}, k_{1}\right)$-continuity. Thus let us now investigate various properties of $\left(k_{0}, k_{1}\right)$-continuity in $C T C$.

Definition 7. In $C T C$, for two spaces $X_{n_{0}, k_{0}}$ and $Y_{n_{1}, k_{1}}$, a function $f: X_{n_{0}, k_{0}} \rightarrow Y_{n_{1}, k_{1}}$ is said to be a $\left(k_{0}, k_{1}\right)$-homeomorphism if

(1) the map $f$ is bijective, and

(2) the map $f$ is a $\left(k_{0}, k_{1}\right)$-continuous map and further, $f^{-1}$ is a $\left(k_{1}, k_{0}\right)$-continuous map.

In this case we say that $X_{n_{0}, k_{0}}$ is $\left(k_{0}, k_{1}\right)$-homeomorphic to $Y_{n_{1}, k_{1}}$.

Obviously, in $C T C, k$-connectedness is $k$-homeomorphic property. Unlike the KD- $k$-homeomorphic properties of Example 3.9, a $\left(k_{0}, k_{1}\right)$ homeomorphism has its intrinsic features as follows.

Theorem 3.11. In $C T C$, let us consider the sets in Figure 3 (see also Example 3.9). Then, we obtain the following.

(1) $\left(X_{1}\right)_{2,8}$ is 8-homeomorphic to $\left(X_{2}\right)_{2,8}$.

(2) $\left(Y_{i}\right)_{3,18}$ is not 18-homeomorphic to $\left(Y_{j}\right)_{3,18}$ if $i \neq j$ and $i, j \in$ $\{1,2,3\}$.

(3) $\left(Z_{1}\right)_{3,26}$ is 26-homeomorphic to $\left(Z_{2}\right)_{3,26}$.

Proof: The proofs of (1) [13] and (3) are trivial.

(2) In $\left(Y_{1}\right)_{3,18}$, since the smallest open set containing the point $x_{2}$ is the set $\left\{x_{0}, x_{1}, x_{2}, x_{3}\right\}$, we obtain that $N_{18}^{*}\left(x_{2}, 2\right)=Y_{1}-\left\{x_{5}\right\}$. Similarly, 
we obtain the smallest 18-neighborhood of $x_{4}$, denoted by $N_{18}^{*}\left(x_{4}, 2\right)=$ $Y_{1}-\left\{x_{1}\right\}$.

Meanwhile, in $\left(Y_{2}\right)_{3,18}$, each point $y_{i} \in Y_{2}, i \in[1,4]_{\mathbf{Z}}$, has $N_{18}^{*}\left(y_{i}, 1\right)$ and the point $y_{0}$ has the smallest 18-neighborhood of $y_{0}, N_{18}^{*}\left(y_{0}, 2\right)=$ $Y_{2}-\left\{y_{3}\right\}$. Thus there is no 18-homeomorphism $h:\left(Y_{1}\right)_{3,18} \rightarrow\left(Y_{2}\right)_{3,18}$.

Next, since the Khalimsky topology of $\left(Y_{3}\right)_{3,18}$ is essential to the discrete topology of $Y_{3}$, neither $\left(Y_{1}\right)_{3,18}$ nor $\left(Y_{2}\right)_{3,18}$ can be 18-homeomorphic to $\left(Y_{3}\right)_{3,18}$.

By Theorem 3.11, we see that $C T C$ gives some benefits in studying a space $X_{n, k}$ from the viewpoint of digital topology. Besides, we obtain the following.

Remark 3.12. (1) In CTC, there is the only simple closed 8-curves with four elements in $\left(\mathbf{Z}^{2}, T^{2}\right)$ up to 8-homeomorphism such as $\left(X_{1}\right)_{2,8}$ in Theorem 3.11.

(2) In $C T C$, there are three types of simple closed 18-curves with 6 elements in $(2 \times 2 \times 1)$-form of $\left(\mathbf{Z}^{3}, T^{3}\right)$ up to 18-homeomorphism such as $\left(Y_{i}\right)_{3,18}, i \in\{1,2,3\}$, in Theorem 3.11 .

(3) In $C T C$, there is the only simple closed 26-curves with four elements in $(2 \times 2 \times 2)$-form of $\left(\mathbf{Z}^{3}, T^{3}\right)$ up to 26-homeomorphism such as $\left(Z_{1}\right)_{3,26}$ in Theorem 3.11.

(4) In CTC, as a generalization of the cases in (1) and (3) above, we observe that there is a simple closed $\left(3^{n}-1\right)$-curves with four elements in $\underbrace{(2 \times \cdots \times 2)}_{n \text {-times }}$-form of $\left(\mathbf{Z}^{n}, T^{n}\right)$ up to $\left(3^{n}-1\right)$-homeomorphism, $n \geq 4$.

Remark 3.13. In view of Theorems 3.10 and 3.11, we observe that $\left(3^{n}-1\right)$-continuity in $C T C$ is not equivalent to $K D-\left(3^{n}-1\right)$-continuity.

\section{Concluding remark and further works}

We have investigated properties of several continuities and homeomorphisms in digital topology which can be used in studying mathematical morphology and the development of covering theory in $C T C$ and $K D T C$ as a further work.

\section{References}

[1] P. Alexandorff, Diskrete Räume, Mat. Sb. 2 (1937) 501-518.

[2] L. Boxer, Digitally continuous functions Pattern Recognition Letters 15 (1994) 833-839. 
[3] U. Eckhardt, L. J. Latecki, Topologies for digital spaces $\mathbf{Z}^{2}$ and $\mathbf{Z}^{3}$, Computer Vision Image Understanding 95(2003) 261-262.

[4] N.D. Georgiou, S.E. Han, Generalized topological function spaces and a classication of generalized computer topological spaces, Filomat 26(3) (2012) 539-552.

[5] S.E. Han, Non-product property of the digital fundamental group, Information Sciences 171 (1-3) (2005) 73-91.

[6] S.E. Han, On the simplicial complex stemmed from a digital graph, Honam Mathematical Journal 27 (1)(2005) 115-129

[7] S.E. Han, Continuities and homeomorphisms in computer topology and their applications, J. Korean Math. Soc. 45 (2008) 923-952.

[8] S.E. Han, Equivalent $\left(k_{0}, k_{1}\right)$-covering and generalized digital lifting, Information Sci. 178 (2008) 550-561.

[9] S.E. Han, The $k$-homotopic thinning and a torus-like digital image in $\mathbf{Z}^{n}$, J. Math. Imaging Vision 31 (2008) 1-16.

[10] S.E. Han, KD- $\left(k_{0}, k_{1}\right)$-homotopy equivalence and its applications, J. Korean Math. Soc. 47 (2010) 1031-1054.

[11] S.E. Han, Extension problem of several continuties in computer topology, Bull. Korean Math. Soc. 47 (2010) 915-932.

[12] S. E. Han, Category which is suitable for studying Khalimsky topological spaces with digital connectivity, Honam Mathematical Journal 33(2) (2011) 231-246.

[13] S.E. Han, Homotopy equivalence which is suitable for studying Khalimsky $n \mathrm{D}$ spaces, Topology Appl. 159 (2012) 1705-1714.

[14] S.E. Han, N.D. Georgiou, On computer topological function space, J. Korean Math. Soc. 46 (2009) 841-857.

[15] Jeang Min Kang, S.E. Han, Compression of Khalimsky topological spaces, Filomat, in press.

[16] E. Khalimsky, R. Kopperman, P.R. Meyer, Computer graphics and connected topologies on finite ordered sets, Topology and Its Applications, 36(1) (1991) $1-17$.

[17] In-Soo Kim, S.E. Han, C.J. Yoo, The pasting property of digital continuity, Acta Appl. Math. 110 (2010) 399-408.

[18] T.Y. Kong, A. Rosenfeld, Topological Algorithms for the Digital Image Processing, Elsevier Science, Amsterdam, 1996.

[19] A. Rosenfeld, Arcs and curves in digital pictures, Jour. of the ACM, 20 (1973) 81-87.

Sik Lee

Department of Mathematics Education, Chonnam National University, Gwangju 500-757, Korea.

E-mail: slee@chonnam.ac.kr 
Sang-Eon Han

Faculty of Liberal Education, Institute of Pure and Applied Mathematics, Chonbuk National University,

Jeonju 561-756, Korea.

E-mail: sehan@jbnu.ac.kr 